\title{
Radiologic, Endoscopic and Functional Patterns in Patients with Symptomatic Gastroesophageal Reflux Disease after Roux-en-Y Gastric Bypass
}

TIInSELSPITAL Borbély Y, Nett P, Kröll D, Moreno P, Tutuian R, Lenglinger J

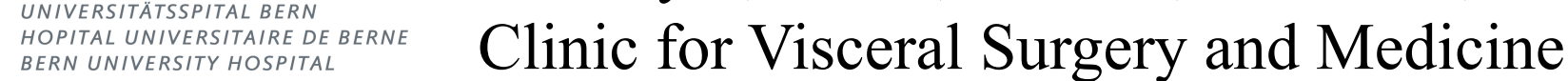

\section{Introduction:}

Roux-en-Y gastric Bypass (RYGB):

- gold standard in treatment of morbid obesity and Gastroesophageal Reflux Disease (GERD)

- Resolution of GERD-Symptoms around 85-90\%.

- So far, data on evaluation for persistent GERD after RYGB is scarce

\section{Methods:}

- All patients evaluated for persistent GERD after RYGB 01/12 - 12/16

- assessed with questionnaires, endoscopy, 24h-pH-impendance-manometry, barium swallows, (MR-Enteroclysis or thoracoabdominal CT)

- All values are medians with minimum and maximum in parentheses

\section{Results:}

- 47 patients, 27 (57.4\%) female. Median age was 36.5 years (min 19.1-max 67.2), interval between initial RYGB and evaluation 3.8y (0.6-12.6)

- BMI@RYGB was 46.3kg/m2 (35.4-65), BMI@evaluation 30.3 kg/m2 (20.3-47.2), total weight loss 34.4\% (14.2-56.7) , excessive BMI Loss 74.6\% (27-123)

\section{Symptoms:}

- typical GERD

- obstructive

- respiratory

- pain

$$
\begin{aligned}
& 44(94 \%) \\
& 18(38 \%) \\
& 8(17 \%) \\
& 21(45 \%)
\end{aligned}
$$

\section{Endoscopic evaluation:}

- Esophagitis (LA grade)

- A

$44(94 \%)$

- B

$8(17 \%)$

- C $2(4 \%)$

- D $3(6 \%)$

- Barrett 7 (15\%)

- Marginal ulcers 4 (9\%)

\section{Radiological evaluation:}

- Pouch-gastric fistula

- Large pouch ( $>6 \mathrm{~cm}$ width) $2(4 \%)$ $5(11 \%)$

- Hiatal hernia - $\quad<2 \mathrm{~cm} 6$ (13\%), 2-5cm $11(23 \%)$, $>5 \mathrm{~cm} 5(11 \%)$, PEH $3(6 \%)$

\section{Functional evaluation:}

- 24h-pH-impedance-metry $(n=44)$

- Abnormal acid exposure $(>4 \% \mathrm{pH}<4) 27(61 \%)$

- $>40$ reflux episodes $30(68 \%)$

- positive symptom association 24 (55\%), functional symptoms $8(18 \%)$

- High resolution manometry $(n=45)$

- Hypotensive LES $26(58 \%)$, aperistalsis 8 (18\%), ineffective motility $9(20 \%)$

\section{Conclusion:}

- evaluation for persistent GERD after RYGB revealed a high percentage of hiatal herniae, hypotensive LES and other severe esophageal motility disorders 